\title{
CUANTIFICACIONES SOCIODEMOGRÁFICAS DE LA CIUDAD DE SANTO DOMINGO EN EL SIGLO XVI $\left(^{*}\right)$
}

POR

ROBERTO CASSÁ

Siguiendo de cerca tres censos se analiza la evolución y desarrollo de la Ciudad de Santo Domingo. Estos recuentos de población $(1528,1586$, 1606), aunque fueron todos ellos exigidos por la administración civil, tienen metodologías diferentes (posesión de armas, vecindario), lo que implica algunas imprecisiones. Desde 1528 la ciudad de Santo Domingo pierde su protagonismo de primera capital hispanoamericana, en beneficio de otras áreas. No obstante, a pesar de su escaso crecimiento demográfico, se multiplican los oficios y se radicaliza el poder (económico, social) de las minorias.

\section{I}

Entre los rasgos tempranos que adoptó la vida de la entonces llamada Isla Española, primera colonia europea en América, figura la tendencia a la centralización de actividades en la

\footnotetext{
SIGLA UTILIZADA:

AGI: Archivo General de Indias, Sevila.

(*) Este texto se ha beneficiado de las observaciones de mi amigo Genaro Rodríguez, investigador en el Archivo General de Indias especializado en el siglo XVI.
} 
ciudad de Santo Domingo. La ciudad concentró el grueso del comercio exterior de la isla, al tiempo que operaba como punto de escala del comercio transatlántico. Tal fenómeno no resultó ajeno a la función que tenía como centro administrativo dentro del naciente imperio español.

Dada la tendencia a la correlación entre funciones administrativas y el acceso a la gestión terrateniente esclavista, la generalidad de los grandes propietarios tenían la ciudad como residencia. La incidencia directa en el aparato público se haría una constante en la clase esclavista; a ello se agregaba la necesaria conexión de los grandes propietarios con el último eslabón local del circuito mercantil. Por otro lado, casas comerciales sevillanas y genovesas, utilizando las ventajas suplementarias de sus posiciones en los circuitos europeos, se involucraron directamente en la producción de azúcar (1).

Más allá de los determinantes económicos en la base urbana del poblamiento, debe tomarse en consideración el conjunto de elementos funcionales que conectaban poder político y riqueza, prestigio y cosmovisión suntuaria. El campo tropical era percibido como un terreno hostil, asociado a faenas duras que se asignaban a esclavos o a libres desfavorecidos. La residencia urbana, por otra parte, se tornaba destino inevitable para aquellos que desempeñaban las posiciones dominantes dentro de las relaciones de producción. El estilo de vida urbano era deseado por la generalidad de blancos peninsulares, al tiempo que las perspectivas de la promoción social sólo podían materializarse en dicho entorno, en base a las relaciones con los negocios y la autoridad.

De manera que la actividad agrícola, dirigida a mercados externos y tornada sostén de la economía urbana desde poco después de 1520, así como los ordenamientos institucionales del Estado castellano, remitían a la imbricación social de los grandes propietarios con la cúspide burocrática y el alto comercio. Desde el punto de vista demográfico y tras superarse la etapa minera, Santo Domingo se estructuró ante todo como

(1) Genaro RodRíguEz, "Esclavitud y vida rural en los ingenios azucareros de Santo Domingo, siglo XVI", Anuario de Estudios Americanos, Sevilla, año XLIX, 1992, págs. 89-117.

R. I., 1996, n. $^{\circ} 208$ 
aglomeración de propietarios agrícolas, en la medida en que así éstos aprovechaban las ventajas que se derivaban de las funciones mercantiles y administrativas de la urbe.

Las categorías de terratenientes, mercaderes y funcionarios tendían a converger y a retroalimentarse en intereses y colaboraciones, no obstante, las pugnas que las dividían. La cúspide del alto comercio, como se observa en las familias de italianos, se inmiscuía en el control de la producción de azúcar. Los hacendados, por su parte, se asociaban con mercaderes. Por último, parecía inevitable que hasta los integrantes de la Real Audiencia se involucraran en actividades económicas, contraviniendo las expresas disposiciones en sentido contrario. Desde tal ángulo, se conjugaban categorías económicas y administrativas con individuos y clanes que rivalizaban por la preeminencia.

Tal relación propendió a la identificación entre Santo Domingo y la vida urbana (2). Santo Domingo se definió con rasgos distintos a los de las villas. Estas aglomeraciones concentraban casi con exclusividad a productores agrarios o pecuarios de las zonas circundantes, y funcionalmente se conformaban como simples extensiones del campo. La división del trabajo en ellas se revela prácticamente inexistente, mientras que en Santo Domingo, como es materia de este artículo, estuvo siempre presente un sector dedicado a actividades no agrícolas.

Adicionalmente a lo que representaban las divisiones del trabajo, únicamente Santo Domingo tenía la fisonomía de ciudad, con mayoría de casas de piedra, calles bien trazadas, aparatos administrativos y servicios, etc. En las villas se llevaba una vida en extremo rústica, entre sembradíos y viviendas de madera y yagua. Ni siquiera los templos de la mayoría de ellas estaban construidos de piedra (3). Con excepción parcial de Santiago, La Yaguana y La Vega, las villas carecían de servicios administrativos (4).

(2) Roberto CASSÁ y Genaro RodRÍGuEZ, «Algunos procesos formativos de la identidad nacional dominicana», Estudios Sociales, Santo Domingo, año XXV, núm. 88, 1992, págs. 67-98.

(3) Américo Lugo, Historia de Santo Domingo, Ciudad Trujillo, 1952, pág. 160 .

(4) Ibid., págs. 211 y sigs. 
La concentración del fenómeno urbano y sus bases de sustento comportaron consecuencias en el fenómeno criollo en el siglo XVI, emergiendo una segmentación espacial en los lineamientos de formación del conglomerado demográfico. En la ciudad hacía eclosión un criollismo de fuerte impronta hispánica, en tanto que, paralelamente, el campo se contraponía como laboratorio de reprocesamiento de lo africano y lo indígena. Entre los estilos de vida de Santo Domingo y las villas para finales del siglo se interponía un fenómeno racial, ya que en las últimas se daba ya cuenta de la condición mulata de la mayoría de sus vecinos (5).

\section{II}

Para discutir los aspectos de lo urbano, se puede acudir a la información cuantitativa disponible. Los recuentos o censos de los pobladores de la ciudad efectuados a lo largo del siglo XVI sirven de materia prima al presente texto. El abordaje de la vida urbana se realiza a través de magnitudes cuantitativas que permiten ubicar relaciones y su evolución; no obstante, no se hace uso de todas las fuentes de ese tipo, sino de aquellas que iluminan aspectos a destacar de las relaciones sociales (6).

La primera de esas fuentes es un recuento de la población masculina adulta, apta para portar armas. Se realizó en 1528 con motivo de la aparición del primer navío pirata en costas americanas, capitaneado por el francés Diego Ingenios (7). Su editor, Enrique Otte, lo califica de censo, «el primero de Santo Domingo y, según parece, de América» (8). Una segunda fuente

(5) Fray Cipriano de Utrera, Historia militar de Santo Domingo, 3 vols., Ciudad Trujillo, 1950-1952, II, pág. 306.

(6) Por ello, se omite lo que puede calificarse como el primer censo disponible de la isla, el Repartimiento de Indios de 1514, efectuado por Rodrigo de Alburquerque. Se encuentra en Emilio Rodriguez Demorizi, Los dominicos y las encomiendas de indios de la Isla Española, Santo Domingo, 1971.

(7) Se encuentra en AGI, Santo Domingo, leg. 9. En esos días apareció el primer navío pirata frente a Santo Domingo. UTRERA [5], I, pág. 251.

(8) Está editado como apéndice en Enrique OTTE: "Carlos V y sus vasallos patrimoniales de América», Clio, Santo Domingo, núm. 116 (enero-junio de 1960), págs. 1-13; el censo está en págs. 14-27.

R. I., 1996, n. 208 
reitera la antecedente, al hacerse un conteo de la población en capacidad de participar en acciones bélicas a resultas de la toma de la ciudad por el corsario Francis Drake en 1586 (9). La tercera ha sido la más comentada, el censo de toda la isla realizado por orden del gobernador Osorio en 1606 con motivo de la despoblación de las comarcas occidentales y septentrionales (10).

El eje del análisis se centrará, pues, en la información censal referida. La consulta de fuentes adicionales contribuye a perfilar aspectos de la vida urbana y a corregir eventuales deficiencias del dato estadístico (11). De todas maneras, se parte del supuesto de que, en lo tocante a cuantificaciones, los censos son más precisos y confiables que las informaciones cualitativas. Como todavía son más seguros los registros seriales, el análisis debió haberse complementado con una fuente capital todavía no explotada desde este ángulo, el Archivo del Arzobispado, que reúne los documentos conservados más antiguos: los registros demográficos de la ciudad amurallada a partir de 1590. En tal caso se hubieran podido intercalar, aun fuera para las últimas décadas de siglo, planos de variaciones continuas (12).

Los dos primeros censos en gran medida son comparables. En varios aspectos, como precisión e identificación del estado de las armas, el censo de 1586 es mejor que el de 1528. Ambos adolecen, ciertamente, de defectos y de sesgos que restringen las posibilidades de examen de variables sociodemográficas. No se trata de censos, en sentido estricto, sino de recuentos de la población masculina adulta libre o con las condiciones étnicosociales para portar armas. Además de las mujeres, niños y la generalidad de ancianos, están ausentes los indios y los escla-

(9) Editado por Emilio Rodríguez DemorizI: «Vecinos de Santo Domingo en 1586 hábiles para las armas», en Relaciones históricas de Santo Domingo, 3 vols., Ciudad Trujillo, 1942-1957, II, págs. 73-108.

(10) Editado por Rodríguez DEMORIZI, ibid., II, págs. 374-445.

(11) Basta referir la importante "Relación de la Isla Española», de 1568, redactada por el lic. Echagoián, en RoDRÍGUEz DEMORIZI, ibid., I, págs. 125-146.

(12) De todas maneras, se toma en cuenta, en los recuentos de profesiones, el examen de dicho registro hecho por Francisco Colom SEvillano, "Noticias de la ciudad de Santo Domingo a fines del siglo XVI (1590-1599)", Clio, año XXVIII, núm. 116 (enero-junio de 1960), págs. 31-53. 
vos; de la misma manera, está ausente la generalidad de la población libre de color, aunque su ligera aparición en el segundo censo entronca con el nudo de los elementos a discutirse.

Estas características impiden llegar a determinaciones cuantitativas exactas, por lo que las magnitudes que se relacionan no pasan de aproximativas, aunque suficiente para los fines del presente análisis. En, los censos de armas el conteo se lleva a cabo a través de hogares. Un hogar, empero, podía contener varios vecinos. No informan casi nada acerca de la porción restante de la población, esto es familiares y otros dependientes de los jefes de familias. No pocas imprecisiones en las unidades de análisis enturbian las posibles inferencias.

El censo de Osorio, en cambio, comprende a todos los vecinos, esto es jefes de familias, incluyendo mujeres, y permite la conexión de la población con las unidades productivas agrarias. Aunque con criterios más laxos y abarcadores que los otros dos, también se restringe a la población libre susceptible de calificar en la condición de vecinos. Por ende, en el censo de Osorio no hay información acerca de los familiares de los vecinos ni de la porción dependiente de los hogares; tampoco incluye a los mulatos y negros libres en el campo, al tiempo de que de seguro excluye a la porción mayoritaria de los libres de color de Santo Domingo y las villas. La cuantía de la masa esclava, todavía mayoritaria, queda proyectada a partir de los trabajadores de los ingenios. Por ende, no hay forma de saber el número de esclavos en la ciudad.

III

Si nos atenemos, aun sea tentativamente, a los conteos que realizan los censos de sujetos aptos para portar armamentos, se puede inferir que la población de la ciudad experimentó un crecimiento moderado. En contraste con las visiones de los informantes de la época, quienes invariablemente advertían una disminución severa del número de vecinos o habitantes, tal constatación ratifica la relativa potencia del fenómeno urbano, como parte consustancial del funcionamiento de la economía esclavista. Cierto que, tras un rápido auge a inicios del siglo y una subsiguiente disminución ligera, se produjo un crecimien-

R. I., 1996, n.o 208 
to lento, hasta desembocar en virtual estancamiento. Gran parte del primer contingente de blancos solteros abandonó la isla, en sucesivas oleadas, cada una más lenta que la anterior: primero a las restantes Antillas, luego a México y, por último, a Perú.

El censo de 1528 contiene un número aproximado de 433 hombres residentes en la ciudad (13), de los cuales unos 281 eran cabezas de hogares (14). En el censo de 1586 se contabilizaron 470 hombres adultos, lo que implica un incremento de $8,5 \%$, suponiendo estricta similaridad de criterios en el conteo (15). Ahora bien, el aumento real de la población debió ser mayor: por un lado, la proporción de jefes de familia, con mujer e hijos, debía ser considerablemente más alta en 1586 que el $65 \%$ de 1528; por otro lado, el tamaño de las familias debió incrementarse en base a una ampliación de la proporción de niños, ancianos y mujeres. Esto se comprueba en la mención que se hace de los hijos: en 1528 aparecen apenas 12, el 2,8 \% de los 433 hombres contabilizados, mientras que en 1586 suman 50, representando el 10,6\%.

La dinámica demográfica de la ciudad parece haber entrado en una fase de estancamiento a finales de siglo, lo que se puede atribuir a la decadencia de la economía esclavista, aunque todavía en esas décadas persistieron factores que coadyuvaban al incremento demográfico en el conjunto de la isla. Por una parte, la población libre tendía a acrecentarse por vía vegetativa en mayor proporción que los libres de antaño -en mayoría solteros- y que los esclavos, que sufrían atroces mortandades y desbalance de sexos. Al mismo tiempo, la emigración estaba

(13) En más de una ocasión no se consigna el número exacto de los dependientes de vecinos prominentes, mencionados como «varios», amén de otras imprecisiones.

(14) La ambigüedad en la formulación se deriva de la naturaleza del censo. En lo adelante se obvia la explicitación de la imprecisión, pero se aclara que todo el análisis atiende a magnitudes aproximadas.

(15) Debe consignarse que, aunque los censos se confeccionaron con metodología similar, el de 1586 no contabilizó los dependientes residentes en el campo. Sin duda, una parte de los blancos registrados en el campo en 1528 tenían relación con la vida urbana, por lo que se precisa un ajuste parcial en la comparación de población entre ambas informaciones. 
fuertemente obstaculizada por el interés de la Corona de mantener una base sólida en Santo Domingo.

De manera que, operando en forma reducida los factores emigratorios, el crecimiento vegetativo de la población urbana únicamente podía quedar neutralizado por la migración interior. La inflexión demográfica se comprueba en el censo de Osorio de 1606, que arroja un estancamiento en la población libre censada de Santo Domingo, si se la compara con la de veinte años antes. Aunque se elaboró con una metodología distinta a los otros dos, en el censo de Osorio da cuenta de la estabilidad del número de vecinos, al arrojar 648 en Santo Domingo, de los cuales se pueden obviar 28 sacerdotes; si se restan las 195 viudas y solteras, todavía quedan 425 hombres jefes de familia, una cifra similar a los aproximadamente 420 hombres que resultan del total de 470 vecinos contados en 1586 tras la sustracción de los 50 hijos o yernos.

Las cifras derivadas de los tres censos, como se ha observado, permiten trazar líneas de la dinámica demográfica. Existen otras cifras, provenientes de estimaciones de funcionarios, las cuales presentan la dificultad de imprecisión y de variables criterios subjetivos. Aun así, la mayoría de dichos estimados se reiteran en la cifra de 500 vecinos (16).

La determinación del total de la población remite a considerar la evolución de las categorías sociales que no se tomaban en cuenta en el recuento de vecinos. En un primer momento el sector más importante fue el de los indígenas, tanto naturales como traídos forzosamente. Posteriormente se conformó una elevada población esclava, que sólo empezó a disminuir en las últimas dos décadas del siglo. Por último, cabe tomar en consideración la sostenida ampliación de los mulatos $\mathrm{y}$, en menor medida, de los negros libres, que normalmente no eran contabilizados como vecinos.

Estos sectores cubrían una proporción considerable de la población total de la ciudad, aunque siempre menor que los ve-

(16) La cifra de 500 vecinos es ofrecida ya por el oídor Echagoián, hacia el 1567, en "Relación de la Ísla Española», pág. 135. Se encuentra reiterada por el visitador Ribero, en 1580; AGI, Santo Domingo 70, ramo 1, doc. 10. La única estimación superior, de 600 vecinos, es la de Álvaro Caballero, en 1541; véase UTRERA [5], II, pág. 206.

R. I., 1996, n." 208 
cinos y sus familiares. Por ejemplo, el visitador Ribero indica que en los límites de la ciudad residían 2.000 esclavos en las casas de sus 500 vecinos. De acuerdo a Grajeda de Guzmán, en la misma época, en la ciudad y en las zonas rurales próximas había 2.000 hombres mestizos y mulatos, aparte de los esclavos y 300 mulatos que trabajaban en las estancias (17). De esas informaciones se infiere que, pese a la preferencia por los criados esclavos, la primacía de la población esclava no se extendiera a la zona urbana, sino que se manifestara de manera únicamente en el campo. Si se siguen las informaciones precedentes, para fines del siglo seguía predominando en la ciudad de Santo Domingo el sector considerado de raza blanca, cercano a 3.000 personas, bajo el supuesto de un per capita de 6 personas por familia. En este período, el sector de mulatos tendió con rapidez a superar al de los negros, lo que se ratifica en las cifras de 2.000 esclavos urbanos y 2.000 mulatos de la ciudad y alrededores. De manera que sobre la base de esas informaciones de finales de siglo, se puede estimar el total de la población de la ciudad en cerca de 7.000 personas.

Entre las fechas extremas, la ciudad transitó de una población libre casi exclusivamente española al predominio franco de los criollos nacidos en la isla. Así, la ciudad, como conglomerado de edificaciones, se fue ampliando a medida que evolucionaba desde una composición mayoritariamente masculina adulta hacia el equilibrio de sexos y de grupos de edad, producto de la estabilización de un grupo poblacional que se radicó en forma definitiva mediante la organización familiar. La generalidad de los libres de 1528, al haber nacido en la península y en el contexto de sus búsquedas aventureras, eran solteros o dejaban sus mujeres en el suelo natal.

\section{IV}

La dialéctica de continuidad y cambio inherente al proceso de criollización comprendía variaciones en la composición de la población que son recogidas en la información contenida en

(17) AGI, Santo Domingo 51, doc. 65. 
los censos. Una de las más significativas es la división de los hombres blancos en propietarios y dependientes, estos últimos designados de varias maneras. En esta segmentación se advierte una herencia de las relaciones sociales presentes en la península a fines del medievo. Por lo visto, una parte considerable de los migrantes y criollos tenían que subordinarse a un propietario -generalmente de considerable fortuna- aunque en teoría tuvieran igual estatus social. El acceso a la tierra en calidad de propietario en forma-progresiva requería de recursos para adquirir esclavos. Los únicos blancos dispuestos a laborar la tierra eran los portugueses.

La disponibilidad de dependientes blancos obedecía a dos principios clave. El primero era de carácter utilitario-económico: como mano de obra auxiliar, fuese para labores que requerían de pericia especializada (como los maestros para hacer el azúcar) o para controlar y dirigir a los esclavos; esta porción de la población se localizaba mayoritariamente en el campo, pues su función económica requería que habitaran en las unidades productivas.

Un segundo principio partía de la valoración suntuaria, a su vez expresión de las aspiraciones aristocráticas de la cúspide de la clase esclavista (18). El prestigio se consolidaba por medio de la disposición de "cristianos», algunos de los cuales quedaban conceptualizados como "criados». El control de tales criados constituía primerísima señal de preeminencia. En correspondencia con la cosmovisión feudal, muchas funciones se llevaban a cabo de manera privada, aunque a veces los criados se sostuvieran con recursos estatales.

Por otra parte, los dependientes podían ser hijos en edad de trabajar, al igual que hermanos y demás familiares; también se mencionan otras personas que no pueden verse exactamente como dependientes, sino como "compañeros». Pero el grueso de tales dependientes tenían por función servir a los cabezas de familia. Varios son señalados como "oficiales» u "obreros», «mozos», «españoles» o «cristianos». Aunque no se utilizara mu-

(18) No pocos de los esclavistas de mayor grado de acumulación adquirían costosos títulos de caballeros de Órdenes Militares. Información de Genaro Rodríguez.

R. I., 1996, n.o 208 
cho la categoría en 1528 , la generalidad de estos últimos caen en lo que se designaba como "criados".

Por ende, el término criado ilumina la condición social genérica de estos dependientes blancos. Los criados no estaban atados por vínculos de sujeción, pues no se reiteraron lazos de tipo señorial. Pero tampoco se trataba de simples asalariados, ya que estaba presente la superioridad formal y moral del patrono. Esa relación de dependencia adquiría faceta extrema en quienes llegaban desde la península traídos expresamente por un funcionario o hacendado; pero también abarcaba a la población blanca no propietaria, que establecía una gama de vínculos con los patronos, desde la relación salarial hasta la que se reforzaba con ayuda del parentesco.

La importancia del fenómeno se advierte en el censo de 1528, que reseña una población dependiente similar, en lo cuantitativo, a la propietaria de hogares.

Cuadro I

CABEZAS DE HOGARES Y DEPENDIENTES, 1528

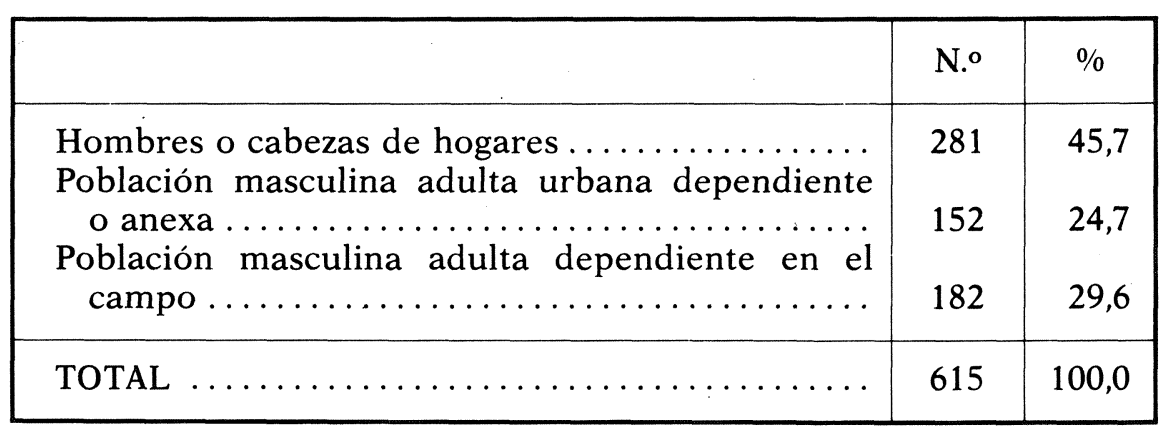

Debe aclararse que mientras los dependientes del campo tendían a ser asalariados de los grandes propietarios, en la ciudad había no pocos familiares o "compañeros» de similar condición social del propietario. De todas maneras, una elevada porción de los dependientes urbanos caían en la condición de asalariados y criados. 
El fenómeno revela la clave articuladora de las relaciones sociales: el dominio ejercido por una fracción reducida de grandes hacendados esclavistas vinculados al control de posiciones en el aparato público. El poder social se expresaba en la elevada proporción de los criados acaparada por unos pocos potentados. De los 182 dependientes en el campo, 117 (el $64 \%$ ) se relacionaban con apenas 10 prominentes personajes, casi todós funcionarios, cada uno de los cuales disponían de por lo menos tres criados urbanos. Quien tenía mayor número de criados era el encomendero Juan de Villoria, con 33, de los cuales 30 en el campo. En la ciudad descollaba la virreina María de Toledo, con 19. Aparte de ellos, sólo unos pocos mercaderes y artesanos tenían algún que otro criado $u$ asalariado urbano.

En la medida en que fue aumentando la población criolla disminuyó la proporción de los dependientes blancos. Y es que la tendencia social subyacente del mundo americano, en cuya química inédita Santo Domingo desempeñaba una función pionera, propendía a la equiparación del blanco con el propietario y del dependiente con el esclavo. Empero, la relación entre patronos y criados no desapareció en la centuria. El componente socio-cultural implicado seguía gravitando, señal de la relativa continuidad de líneas de estructuración de las relaciones sociales. Pero en el censo de 1586 los dependientes pasaron a representar una franca minoría de la población. De un total de 470 personas censadas, los que figuran como dependientes se pueden dividir en las categorías desglosadas en el siguiente cuadro.

La elevada participación de hijos y otros familiares contribuyó a disminuir la porción de otros dependientes, minimizando su peso en el conglomerado. Aunque no se consigna en forma textual, el grueso de los 57 dependientes mencionados de formá genérica (como españoles, cristianos, etc.) caen en la misma condición de los calificados como criados. Esto arroja un total de criados de alrededor de $19 \%$, bastante menor que el porcentaje correspondiente a medio siglo antes: entonces, sólo tomando a la población masculina de la ciudad, los dependientes representaban el $35 \%$ del subtotal, como se observa en el cuadro II; en cualquier caso, resulta concluyente que de alrededor de $45 \%$ los cabezas de hogares hayan pasado a $67 \%$, proporción que en realidad todavía cabe expandir por la 
ampliación del número de viudas y otras mujeres cabeza de familia.

\section{Cuadro II}

TIPOS DE DEPENDIENTES, 1586

\begin{tabular}{|c|c|c|}
\hline & N.o & $\%$ \\
\hline Hijos, yernos o sobrinos ..... & 50 & 10,6 \\
\hline Hermanos, cuñados o primos & 17 & 3,6 \\
\hline Criados $\ldots \ldots \ldots \ldots \ldots \ldots \ldots$ & 32 & 6,8 \\
\hline Otros dependientes de condición no consignada & 57 & 12,1 \\
\hline Subtotal dependientes $\ldots \ldots \ldots \ldots \ldots \ldots \ldots$ & $156(19)$ & 33,2 \\
\hline TOTAL & 470 & 100,0 \\
\hline
\end{tabular}

La disminución de la proporción de dependientes blancos se debió a la doble incidencia de la lógica de reproducción de la economía esclavista y la posición distinta de los blancos en ella. Las funciones accesorias de los propietarios pasaron a ser desempeñadas paulatinamente por libres de color residentes en el campo o, sobre todo, por esclavos. Fue el caso de los maestros de azúcar, quienes en la segunda mitad de siglo dejaron de ser canarios, para predominar los esclavos. La minimización de los criados rurales formó parte de la paulatina deserción de la población blanca de las zonas rurales. Igual de importante resultó la disminución en el número de blancos dependientes o vecinos libres para fines suntuarios.

(19) Por razones variadas, el número restante de no dependientes no coincide con el de cabezas de hogares, pero la diferencia es de apenas 6 personas, nula desde el punto de vista de la significación de las magnitudes. 
Se ha visto que la estructura de la clase de hacendados esclavistas requería del control del aparato administrativo. A ese respecto, se establecía una jerarquía informal pautada por la influencia sobre los organismos de autoridad. Quienes detentaban elevadas posiciones se lucraban en forma primitiva, cometiendo abusos en abierto perjuicio de otros. Fue lo denunciado acerca de Álvaro Caballero, "el más rico de aquella tierra... con grandes delitos... (y) de sus deudos y criados» (20).

La conexión de lo urbano con el aparato administrativo se observa a propósito del peso que tenían algunas funciones. Varios de estos especialistas desempeñaban funciones en la Audiencia, pero era más bien en el entorno del Cabildo donde se daba cita institucional el dominio social. Sobresale del examen de los censos la relativa continuidad de la estructura de cargos y profesiones, en gama tan variada como regidores, alcaldes, secretarios, escribanos, procuradores, abogados, alguaciles, miembros del Cabildo Eclesiástico, etc.

Un examen de las personas que ocupaban estos cargos revela una circulación familiar casi incesante. Los hacendados más ricos y prestigiosos fundaban mayorazgos (21). Los integrantes de esas familias tendían a relacionarse mediante matrimonios de conveniencia, como se observa en la fusión de mayorazgos (22). Las alianzas matrimoniales se focalizaban entre los hacendados y los funcionarios, que incluían a una porción mayoritaria de peninsulares (23). Se conformaron redes familiares, expresadas en la proclividad al matrimonio entre primos de diversos grados, lo que daba lugar a líneas de confluencia entre instancias administrativas que normalmente rivalizaban (24).

(20) Echagoián, «Relación de la Isla Española» [11], pág. 140.

(21) Para un caso, cfr. Fray Cipriano de Utrera, Don Rodrigo de Bastidas, Santo Domingo, 1930.

(22) Información de Constancio Cassá. Véase Fray Cipriano de UtRERA, «Epigrafía colonial (1-6)», en Santo Domingo: Dilucidaciones históricas, Santo Domingo, 1995, págs. 499-614.

(23) Emilio Rodriguez Demorizi, Familias hispanoamericanas, Ciudad Trujillo, 1958.

(24) Genaro Rodríguez, "Las luchas políticas en Santo Domingo en el siglo XVI", Inédito.

R. I., 1996, n." 208 
Pese a la dependencia de inserción en circuitos mercantiles, en los recuentos cuantitativos revisados se advierte una pobre presencia de los comerciantes profesionales. En el censo de Osorio apenas se contabilizaron 13 mercaderes, 15 tratantes y 10 tenderos, distribución que sugiere el predominio de los comerciantes de pequeña escala. Las comparaciones de cifras indican la disminución del peso de los mercaderes, a tono con las tendencias desfavorables que se derivaban del incremento de las contradicciones económicas del imperio español.

Se podría concluir que la ciudad no se encontró en condiciones de explotar las ventajas relativas que podía deparar a la colonia la mayor antigüedad de establecimiento de los españoles y su posición de eslabón en los circuitos de navegación hasta aproximadamente 1560 . Aunque se registraron asociaciones de hacendados y mercaderes alrededor de negocios con Tierra Firme y, en contados casos, con la península, en términos generales se consolidó el deslinde entre comerciantes locales y hacendados. Pero, a diferencia de las economías maduras de plantación, los primeros normalmente lograban un nivel de acumulación menor que los últimos.

La economía insular se encontró a expensas de los mercaderes metropolitanos, quienes, tenían medios para imponer términos duros a sus clientes. La falta de consistencia de los hacendados, quienes se evidenciaron incapaces de sortear el contexto, formó parte del cuadro de factores que condenó a sucumbir la economía esclavista. Medidas sucesivas, como la prohibición de la navegación desde Galicia o Canarias, en los años 60, tuvieron consecuencias devastadoras sobre la economía insular (25). La respuesta a esa situación no podía ser sino el incremento del contrabando en la porción occidental de la isla. Ahora bien, dadas sus características, el comercio clandestino colidía con la formación de una clase local de mercaderes y, por lo tanto, se interponía con la reproducción de la economía esclavista.

Más estable y relativamente importante puede juzgarse la formación de una clase de productores artesanos. Su presencia se derivaba de las funciones urbanas en aquel contexto.

(25) Marino INCHÁUSTEGUI, Reales cédulas y correspondencia de gobernadores de Santo Domingo, Madrid, 1958, 5 vols., II, págs. 560 y sigs. 
Parece que los artesanos locales podían competir con los productos importados, al aprovechar el bajo precio de materias primas, la débil capacidad importadora y las trabas adicionales que provenían de la distancia con la metrópoli. Por ello, a lo largo del siglo puede identificarse una estructura simple de profesiones, que se advierte en los tres censos así como en los registros de la catedral. En el censo de 1606, el más completo, sobresalen los siguientes oficios artesanales: 20 sastres, 16 carpinteros, 14 zapateros, 14 plateros, 8 panaderos, 4 herreros y 4 albañiles. Aparecen normalmente otros oficios, como candeleros, torneros, barberos, pintores, espaderos, olleros, sederos, canteros y silleros.

\section{VI}

La preponderancia de la oligarquía de hacendados no estuvo exenta de dificultades. Su modus operandi tendía a la exclusión de los restantes blancos, uno de los determinantes de la renovación intermitente de las emigraciones. Por ello, las fuentes registran luchas contra la cúspide burocrática de parte de los pequeños esclavistas y demás blancos libres. Es probable que en ello incidiera la incapacidad de la minoría oligárquica para trascender las trabas que obstaculizaban la consolidación de su poder social. En sentido inverso, esos factores debieron incidir en que la unidad económica denominada estancia, consistente en esclavitud a pequeña escala, presentara ventajas sobre el que se manifestaba en el azúcar. Mientras se producía la decadencia acelerada de la exportación de azúcar, en las últimas dos décadas del siglo, florecían los esquemas alternativos: el hato ganadero en las comarcas occidentales y las estancias en dirección oriental desde Santo Domingo.

Los propietarios de estancias y hatos eran casi siempre vecinos, esto es, blancos o supuestos como tal. En el censo de Osorio se observa que numerosos vecinos de Santo Domingo poseían hatos y estancias de jengibre y alimentos. Sólo los propietarios de los 95 hatos y las 107 estancias contabilizados en la demarcación de Santo Domingo equivalen a la tercera parte de sus 620 vecinos, a lo que deben agregarse los hatos y estancias que los vecinos de la ciudad tenían en las villas. 
Aunque los magnates -casi siempre propietarios de varias unidades- se cuentan entre hateros y estancieros, representaban una minoría dentro del total. A la generalidad de pequeños y medianos esclavistas se deben sumar los artesanos y demás especialistas urbanos, como se ha visto arriba en número relativamente importante. En 1606 se contabilizaron unos 95 individuos susceptibles de ser catalogados como artesanos. El censo de Osorio muestra que, aunque algunos artesanos tenían hatos o estancias, no era lo normal, convalidando el deslinde en esa escala social entre actividades agrícolas y tareas urbanas especializadas.

De todas maneras, estos sectores cubrían en conjunto lo que podría calificarse como tendencia a la equiparación de la población alrededor de patrones delimitados de ingresos. La codificación de las informaciones contenidas en los censos de armamentos permite establecer comparaciones al respecto. El armamento se utiliza como indicador, pues su posesión implicaba una combinación de ingreso y restantes componentes de la condición social.

En primer lugar, se palpa que quienes ostentaban la categoría de vecinos en su mayoría se encontraban por encima de una pobreza miserable, al tiempo que el acceso a la cúspide del poder social estaba harto restringido a pocos. La comprobación de la hipótesis requiere considerar las modificaciones acaecidas en el lapso de medio siglo en el patrón del armamento; a ello se agrega que el censo de 1586 tiene más precisión y contiene mayor número de variables.

En función de esta distribución se puede derivar que vecinos y dependientes estaban concebidos como comunidad guerrera. Es notable el escaso número de individuos carentes de armas. Al no existir un cuerpo militar profesional, las categorías de vecino y combatiente en teoría eran intercambiables (26). De todas maneras, no se concedía efectiva atención a los asuntos de guerra. Constantemente se hacían exigencias generales al respecto, tras constatarse el estado inerme de la ciudad.

(26) Utrera, Historia militar, [5], II, págs. 35 y 36. 
ESTRATIFICACIÓN DE LA TENENCIA DE ARMAS, 1528

\begin{tabular}{|c|c|c|}
\hline & N.o & $\%$ \\
\hline$\ldots \ldots \ldots \ldots \ldots \ldots \ldots \ldots \ldots$ & 101 & 16,5 \\
\hline Lanza .......... & 22 & 3,6 \\
\hline Rodela u otra arma. & 15 & 2,5 \\
\hline Espada $\ldots \ldots \ldots \ldots \ldots$ & 174 & 28,4 \\
\hline Espada y otra arma ........ & 185 & 30,2 \\
\hline Caballo y armas .......... & 87 & 14,2 \\
\hline Escopetas y otras $\ldots \ldots \ldots$ & 28 & 4,6 \\
\hline$\ldots \ldots \ldots \ldots \ldots \ldots \ldots \ldots \ldots \ldots \ldots$ & 612 & 100,0 \\
\hline
\end{tabular}

De la revisión de los no armados, sobresale que muchos de ellos son dependientes, aunque de la misma manera algunos de éstos, sobre todo vinculados a figuras prominentes, se cuentan entre los mejor armados. Lo más importante es que implícitamente había una situación característica de los vecinos en lo que respecta al tipo de armamento. Frente a los extremos de los desarmados o los dotados de caballos y escopetas, con una participación relativa de alrededor del $16 \%$ cada grupo, el grueso de los vecinos tería tan solo algún arma blanca, fundamentalmente una espada.

Estas proporciones se ratifican en el censo de 1586, susceptible de una codificación más precisa.

En el lapso de los 58 años transcurridos entre ambos censos apenas aumentó la proporción de armados, pero queda patente una variación de la calidad del armamento. Esto puede atribuirse a la generalización del arma de fuego y a la creciente amenaza de ataques piratas. Por otra parte, se puede constatar una sensible disminución del número de caballos, lo que revela la preferencia por el arma de fuego, cuyo uso excluía la anterior función dirigente de la caballería. Frente al $16 \%$ en 1528 , en el segundo censo tan solo se registra un 4,7\% de caballeros o supuestos a serlo, fuera por prestigio o ingreso. No obstante 
en 1586 la posesión de caballos para el combate se asociaba más que antes al reducido estrato superior o sus criados.

En el censo de 1586, más significativo que el arma portada resulta lo que la Real Audiencia ordena que se adquiera o mejore. La tendencia central se registra alrededor de quienes tienen escopeta o se les ordena hacerlo, respectivamente 32,1 y $28,6 \%$ del total de vecinos. Quienes no tienen armas y no se les ordena adquirir ninguna son a menudo explícitamente calificados como pobres.

\section{CUADRo IV}

ESTRATIFICACIÓN DE LA TENENCIA DE ARMAS, 1586

\begin{tabular}{|c|c|c|}
\hline & N.o & $\%$ \\
\hline Sin armas. No se ordena nada & 52 & 11,4 \\
\hline Sin armas. Se ordena espada .. & 29 & 6,4 \\
\hline Sin armas. Se ordena escopeta & 100 & 22,0 \\
\hline Sin armas. Se ordena caballo . & 1 & 0,2 \\
\hline Con armas blancas. No se ordena más . & 78 & 17,1 \\
\hline Con armas blancas. Se ordena escopeta & 28 & 6,2 \\
\hline Con armas blancas. Se ordena caballo .. & 2 & 0,4 \\
\hline Con arcabuz o escopeta. No se ordena más & 142 & 31,2 \\
\hline Con arcabuz o escopeta. Se ordena algo más & 4 & 0,9 \\
\hline Con arcabuz o escopeta. Se ordena caballo .. & 1 & 0,2 \\
\hline Con caballo. Se ordena algo más ........... & 4 & 0,9 \\
\hline Con caballo. No se ordena más..... & 14 & 3,0 \\
\hline TOTAL & 455 & 99,9 \\
\hline
\end{tabular}

\section{VII}

Únicamente el censo de 1606 permite obtener detalles acerca de la composición familiar de los vecinos, como se observa a continuación: 


\section{Cuadro V}

TIPOS DE CABEZAS DE FAMILIA, 1606 (27)

\begin{tabular}{|c|c|c|}
\hline CATEGORIAAS & N. ${ }^{\circ}$ & $\%$ \\
\hline Hombres con mujer y familia & 284 & 52,8 \\
\hline Viudos $\ldots \ldots \ldots \ldots \ldots \ldots$ & 13 & 2,4 \\
\hline Viudas $\ldots \ldots \ldots \ldots \ldots \ldots$ & 102 & 19,0 \\
\hline Hombres con familia o hijos & 70 & 13,0 \\
\hline Mujeres con familia o hijos .... & 69 & 12,8 \\
\hline TOTAL & 538 & 100,0 \\
\hline
\end{tabular}

Desde el punto de vista de la tendencia demográfica, lo más importante es que prácticamente todos los vecinos, mujeres y hombres, son señalados con familias, incluidos los viudos y viudas. De los vecinos faltantes, hasta los 620 no religiosos, 37 son personas de color, casi todas con familia.

Igualmente, sobresale la relativa alta proporción -26\%-de los cabezas de hogares, tanto hombres como mujeres, que tienen familia pero no cónyuge, lo que por derivación significa que viven solos o bien en relación de concubinato. A causa de ello, se escenificaron conflictos entre Audiencia y Cabildo Eclesiástico, al rechazar los funcionarios laicos la pretensión del clero de que se persiguiera a amancebados y excomulgados (28).

Al cuadro anterior deben agregarse los vecinos catalogados como no blancos, contabilizados aparte en el censo, viéndose que entre ellos la relación familiar tiene normalmente un carácter más informal que entre los conceptualizados como blancos. El hecho de que ya se contabilizaran 37 vecinos de color,

(27) Como no se dispone del conocimiento de la situación familiar de todos los vecinos, se establecen las proporciones únicamente dentro del conglomerado abarcado.

(28) UTRERA, Historia militar, [5], II, pág. 205. 
en mayoría mujeres solteras, y otras informaciones -como el acceso a la propiedad de esclavos por parte de dos mulatas- revelan que los supuestos excluyentes del régimen esclavista se hallaban vulnerados en la práctica. La inclusión de negros y mulatos en la condición de vecinos no era, por lo demás, un fenómeno iniciado en años recientes. Ya existía por lo menos veinte años atrás, como se observa en unos cuantos hombres de color incluidos en el censo de 1586, momento en que apenas se había iniciado el proceso de decadencia de la industria azucarera. Por lo tanto, la transgresión del patrón normativo de las relaciones sociales debió constituir un fenómeno temprano, al menos en sus primeras expresiones. Las fuentes reiteran las relaciones maritales de esclavistas con sus esclavas, la subsiguiente proliferación de mulatos y su nacimiento en libertad por voluntad del padre propietario.

La dinámica subyacente se contraponía a la reproducción indefinida de la esclavitud, máxime cuando fallaba la trata negrera. La información censal convalida que el sistema esclavista de Santo Domingo contenía peculiaridades que lo diferenciarían de las sociedades de plantación que se desarrollaron en la región a partir del siglo xvir. Y es notable que dichos procesos cobraran cuerpo en la ciudadela de la aristocracia burocrática.

The evolution and development of the city of Santo Domingo are analysed on the basis of three population censuses $(1528,1586$ and 1606). Though all of them were imposed by the civil government, there is a lack of precision due to the fact that they follow different methodologies (possession of weapons, residence). As from 1528 the city of Santo Domingo loses the rank of first Spanish American capital. But despite the city's low demographic growth the oficios (crafts) increase in number and there is also an increment of the social and economic power of the minorities. 